\section{Imaging Multilayers of Ice with Scanning Tunneling Microscopy}

\author{
Konrad Thürmer and Norman C. Bartelt \\ Sandia National Laboratories, Livermore, CA \\ bartelt@sandia.gov
}

Ice plays an important role in many naturally occurring phenomena. For example, most rainfall in temperate climates is triggered by the nucleation of ice around $\mu \mathrm{m}$-sized particles in atmospheric clouds. Another reason to be interested in ice is that thin supported ice films provide excellent model systems for studying the interaction of water with solid surfaces. Despite the importance of water-solid interactions for catalysis, corrosion, water purification and fuel cells, even the basics of this interaction are poorly understood. In fact, the best theoretical models often fail to predict even the simplest phenomena [1]. A fundamental question, for example, is whether water/ice wets a given substrate, i.e., whether the substrate is covered up by a uniformly thick water/ice film. (In the case of non-wetting an ice film would break up into separate three-dimensional (3D) crystallites of varying height, exposing the substrate.) a)

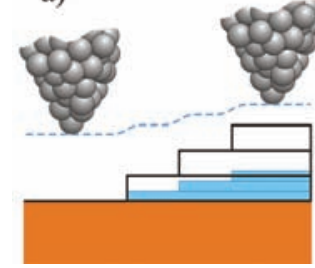

b)

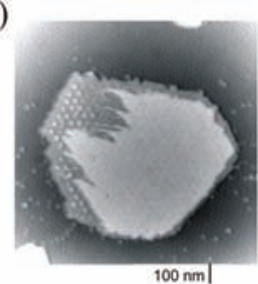

c)

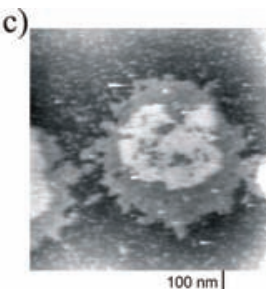

Fig. 1. a) Schematic illustrating the relationship between the real thickness of ice films and the apparent height seen by an STM. As a consequence, the STM tip is closer to the surface above thicker portions of the ice film. b) The STM image shows a $3 \mathrm{ML}$ high crystal surrounded by a $1 M L$ thick wetting layer. Due to a locally stronger tip-sample interaction the $3 \mathrm{ML}$ thick region of the film is damaged. c) 4-5 ML high crystal is damaged even more severely despite using an extremely low tunneling current of $0.4 p A$.

Addressing this question could be straightforward if nondestructive imaging of water/ice films were possible. The capability of Transmission Electron Microscopy (TEM) [2], Scanning Electron Microscopy (SEM) or Low Energy Electron Microscopy (LEEM) in studying ice are very limited, because these techniques use electrons, which quickly damage the ice. X-ray topography, which proved powerful in studying dislocations in ice [3], lacks the nm-resolution necessary to study the initial stages of film growth. Since the 1990's Scanning Tunneling Microscopy (STM) has been used successfully to investigate monolayers of ice on various solid substrates $[1,4,5]$. However, the wetting behavior can change dramatically with films thickness. Often, the first one or few monolayers of water wet the substrate. Then, the growth mode switches and the subsequent water aggregates into 3D clusters of bulk ice. Therefore, to capture the full wetting behavior it is necessary to image multilayerthick ice films.

The difficulty of using STM for ice multilayers comes from its reliance on tunneling electrons, which the nominally insulating ice cannot supply under typical imaging conditions. The consequence of this is illustrated in Fig. 1. While the STM tip seeks to maintain the set tunneling current between tip and sample, the gap between tip and sample surface becomes smaller with increasing film thickness. At $3 \mathrm{ML}$ thickness the STM already starts to interact destruc-
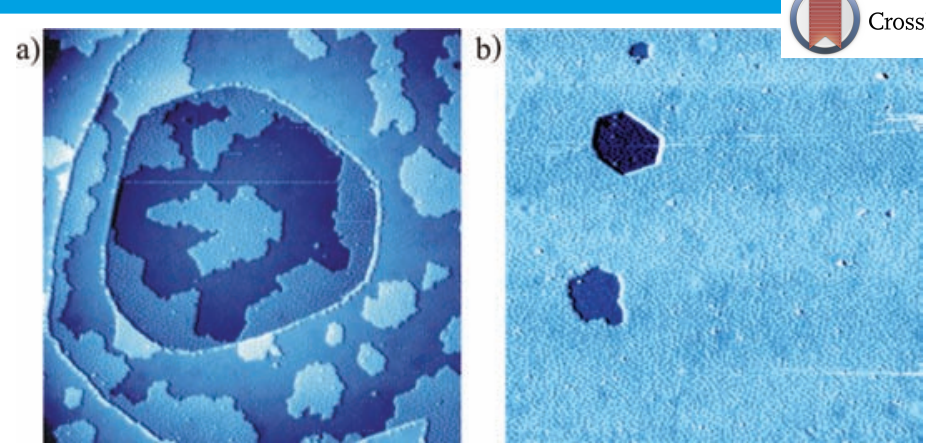

Fig. 2. $150 \mathrm{~nm} \times 150 \mathrm{~nm}$ STM images of the one-molecule-thick wetting layer of (water) ice on Pt(111). a) at 40\% coverage, and b) at $95 \%$ coverage.

tively with the ice film. Even when using an extremely low tunneling current of $0.7 \mathrm{pA}$ the STM tip scrapes away the uppermost bilayer of the ice crystal. For 4-5 bilayer thick film regions, the damage is more severe, as illustrated in Fig 1c. Further attempts at acquiring an image results in more of the film material scattered across the surface and all traces of the original structure lost. Under conventional imaging conditions, it is virtually impossible to obtain any useful information about ice films thicker than $5 \mathrm{ML}$.

We discovered that this difficulty could be overcome by applying negative sample voltages $\mathrm{V}_{\text {sample }}<-6( \pm 1) \mathrm{V}$. As we propose in Ref. [6], this sample bias lifts the electronic states of the ice high enough that the STM tip can extract the needed tunneling electrons from the ice film's valence band. In Ref. [6] we further argue that the high polarizability of ice is a precondition for this electron-extraction process. We found that combining negative sample voltages with tunneling currents lower than $0.5 \mathrm{pA}$ allows nondestructive imaging of up to $\sim 30 \mathrm{ML}$ thick ice films, opening the door to detailed exploration of ice-multilayer growth. We now illustrate the power of this approach by discussing the growth of ice films on a $\mathrm{Pt}(111)$ substrate at $140^{\circ} \mathrm{K}$.

Our STM experiments were performed in an ultrahigh vacuum chamber with a base pressure of $<3 \times 10^{-11} \mathrm{mbar}$. The ice films were grown at a rate of $1 \AA \AA \mathrm{min}$ by directing water vapor onto a cooled $\left(\mathrm{T}=140^{\circ} \mathrm{K}\right)$ platinum sample. During water deposition, the STM tip was retracted far away from the sample to exclude tip-induced modifications of the film growth. The sample was then cooled even further to the imaging temperatures between 100 and $120^{\circ} \mathrm{K}$. As usual for STM images, the surface-height information is contained in the color tone of the images. Higher surface features, i.e., protrusions, appear bright, and depressions dark.

The sequence of STM images in Figs. 2-7 shows how the morphology of a crystalline ice film grown at $140^{\circ} \mathrm{K}$ evolves with increasing (mean film) thickness. Initially the deposited water spreads
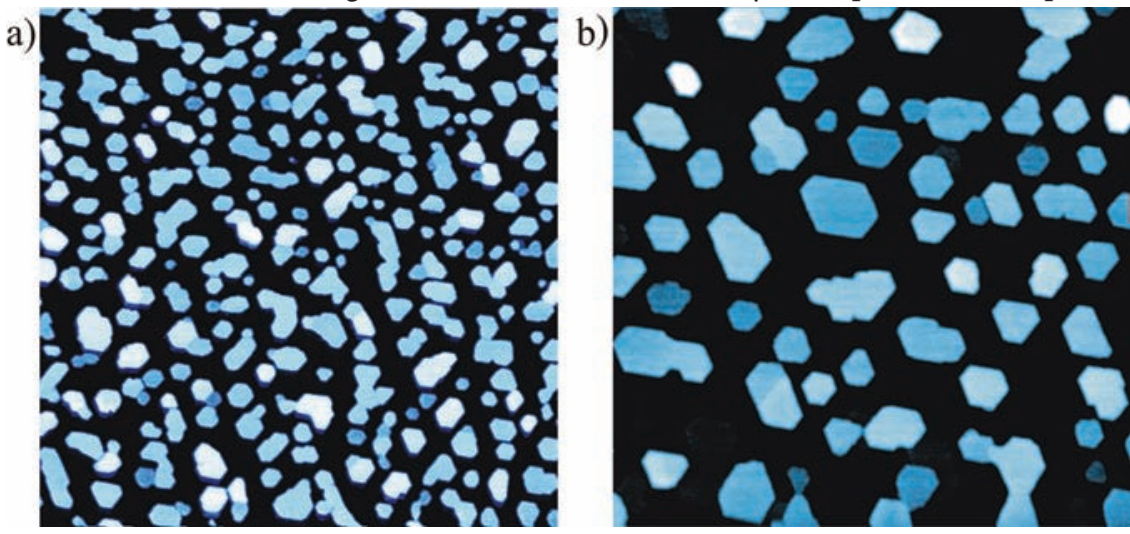

Fig. 3. Crystalline ice/Pt(111) at $1 \mathrm{~nm}$ average film thickness. The field of view is a) 1000 $\mathrm{nm} \times 1000 \mathrm{~nm}$. b) $500 \mathrm{~nm} \times 500 \mathrm{~nm}$. 


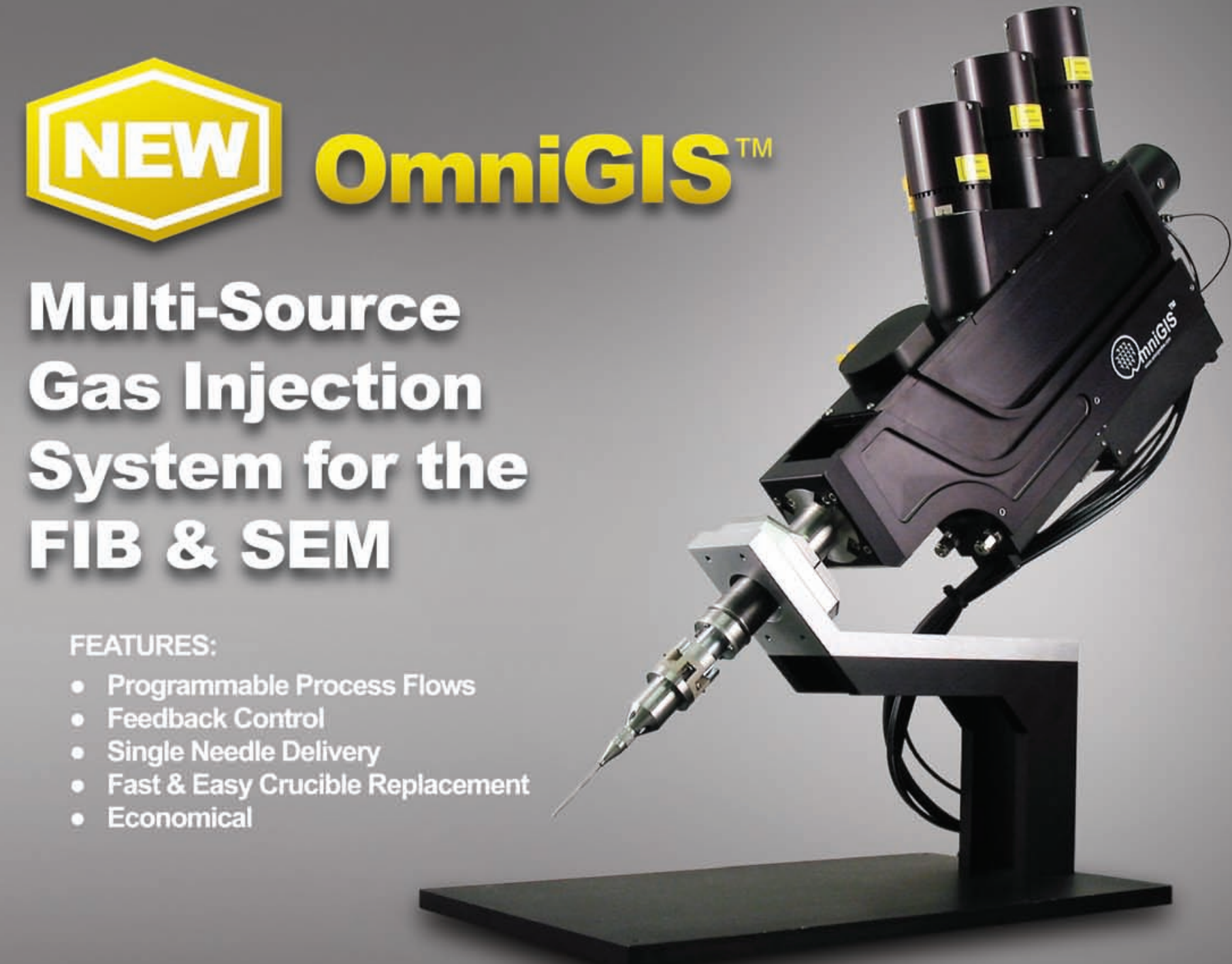

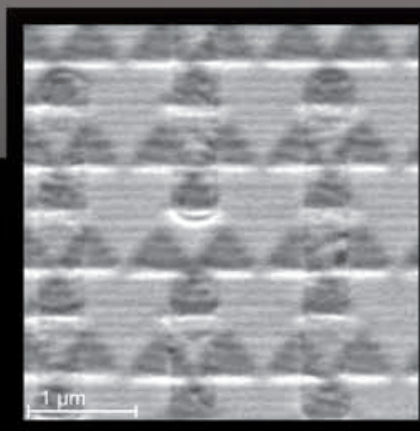

\section{Make}

FIB deposited plasmonic nanostructures

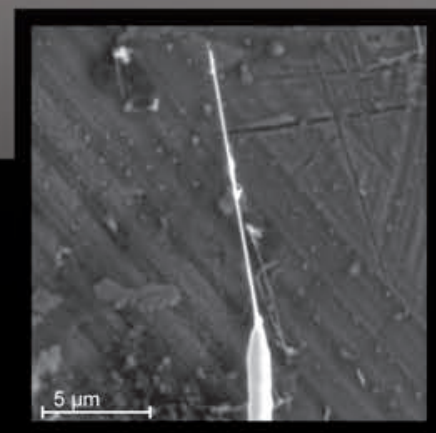

Move

Single nanowire analysis

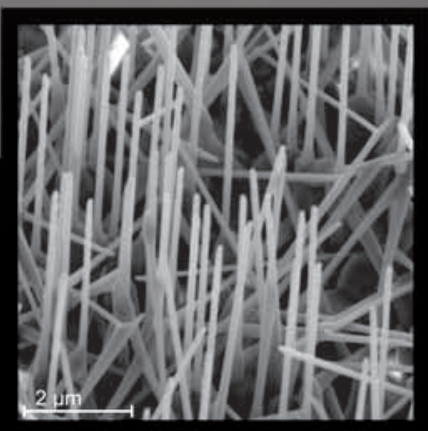

Modify

Chemical surface

alteration

For more information visit us at http://www.omniprobe.com or call us at $\mathbf{2 1 4 . 5 7 0 . 6 8 0 0 .}$

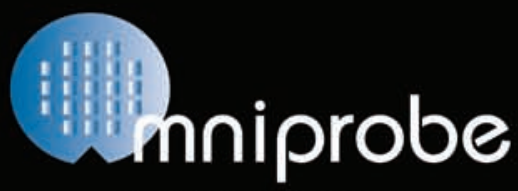




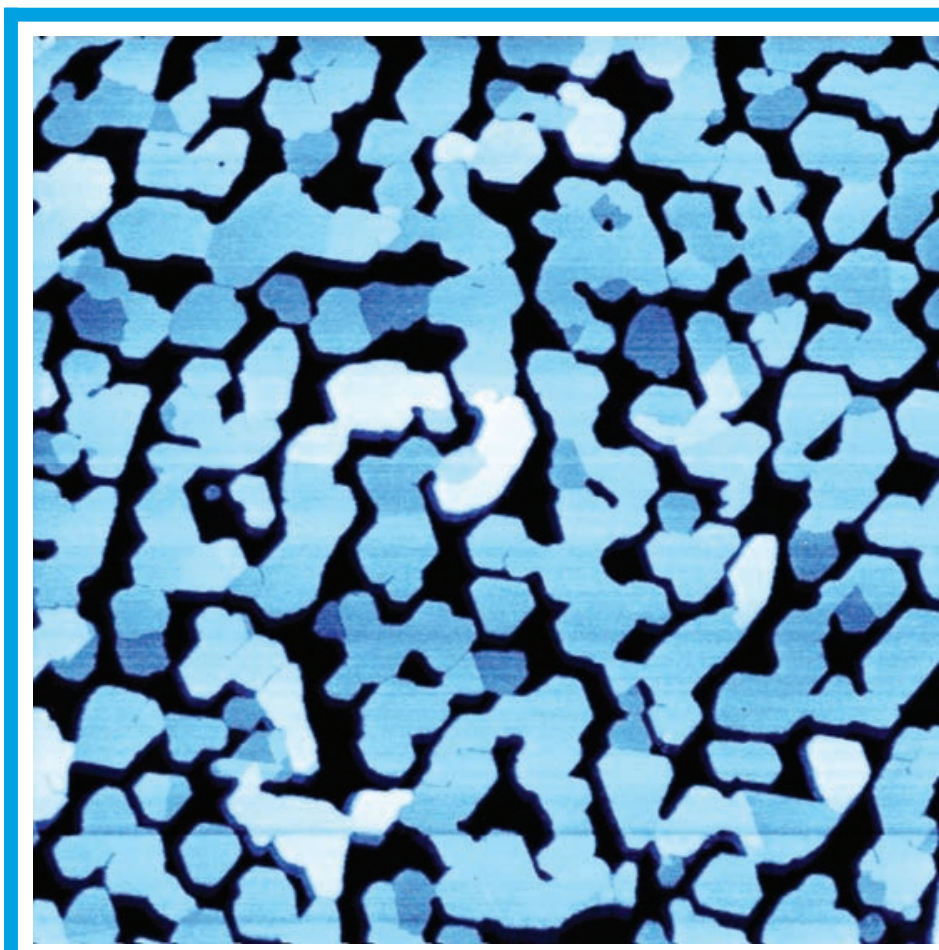

Fig. 4. Field of view: $1000 \mathrm{~nm} \times 1000 \mathrm{~nm}$. Mean film thickness: $1.5 \mathrm{~nm}$.

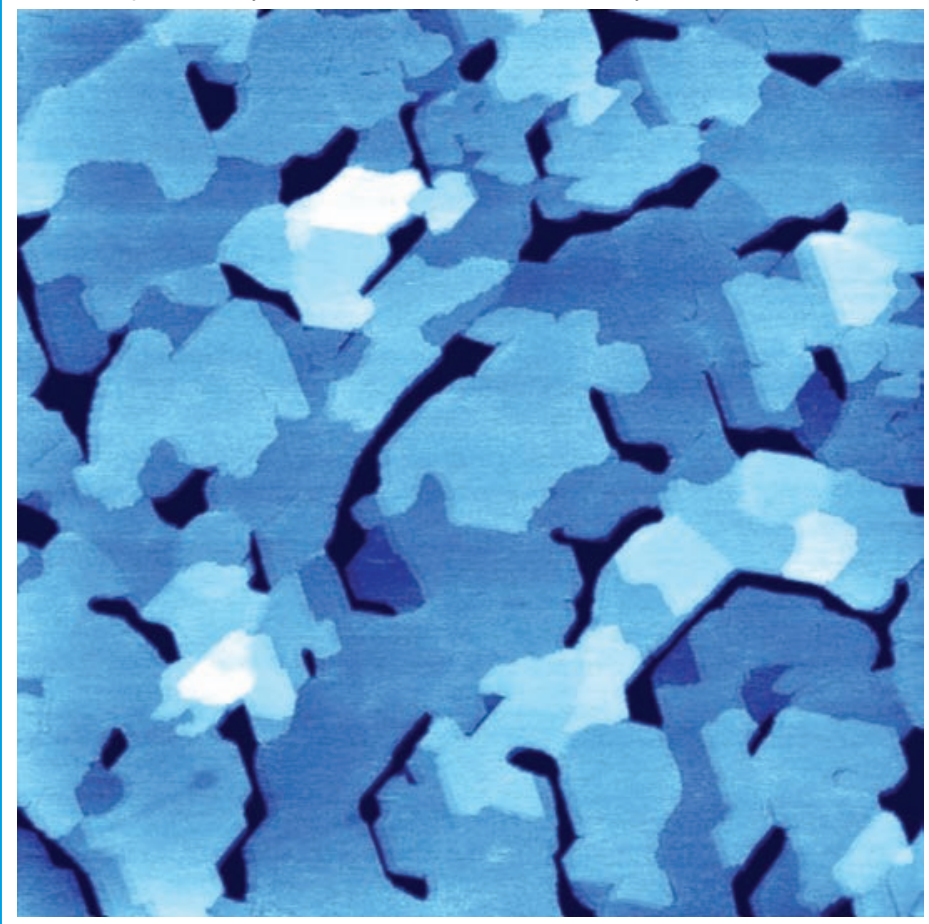

Fig. 5. Field of view: $1000 \mathrm{~nm} \times 1000 \mathrm{~nm}$. Mean film thickness: $4 \mathrm{~nm}$.

out into a two-dimensional wetting layer, which is only one water molecule thick. Fig. 2(a) shows this wetting layer forming irregular patches, which cover $\sim 40 \%$ of the Pt surface. The round slightly triangular loop and the bright line to the left are atomic-height surface steps of the Pt substrate. These lines appear bright because water has attached to the upper side of these steps. Upon depositing more water the one-molecule-thick wetting layer has spread and now covers $\sim 95 \%$ of the surface area (Fig. 2b). The upper, hexagonal dark patch is a one-atom-deep depression in the Pt substrate covered with the wetting layer. The lower, irregular patch is a remaining hole in the wetting layer where bare $\mathrm{Pt}$ is exposed. The rough appearance (corrugation) of the wetting layer reveals that the water molecules are not perfectly arranged.

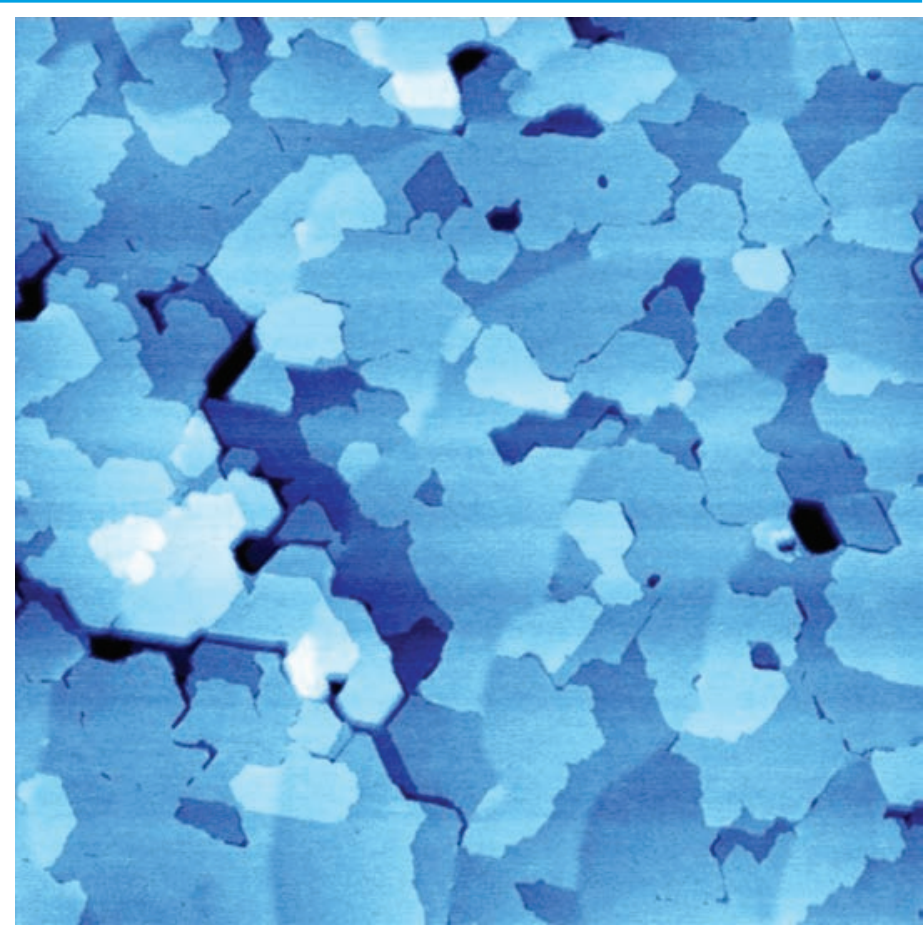

Fig. 6. Field of view: $1000 \mathrm{~nm} \times 1000 \mathrm{~nm}$. Average film thickness: $9 \mathrm{~nm}$.

After the wetting layer has completely covered the Pt substrate, the growth mode switches and three-dimensional crystallites start to form. Fig.3(a) shows a $1 \mu \mathrm{m}^{2}$ and (b) a $500 \mathrm{~nm} \times 500 \mathrm{~nm}$ surface region featuring 2-3 $\mathrm{nm}$ high crystals surrounded by the wetting layer, which now appears black. The crystallites are hexagonally shaped with their edges parallel to the crystallographic $\{11-2\}$ directions of the Pt substrate. This shape suggests that the top facet of the crystallites consist of the basal plane and the side facets being the prismatic faces of the crystalline ice $1 h$ structure.

Upon deposition of additional water-the average film thickness has now reached 4 molecular layers or $1.5 \mathrm{~nm}$ - all crystallites merge into a labyrinthine pattern (see Fig. 4). The dark channels extend down to the wetting layer. At a total film thickness of $4 \mathrm{~nm}$ (see Fig. 5) most channels are filled in, leading to larger, up to $200 \mathrm{~nm}$ wide, atomically flat terraces. Patches of different height levels are separated by ice surface steps, which are often just one or two water molecules high. At a total film thickness of $9 \mathrm{~nm}$ (see Fig. 6) the entire area is covered by an ice film of rather uniform thickness. Just a few pinholes reaching down to the wetting layer remain.

Using the novel non-destructive mode of STM operation, this rich ice-film morphology has now been revealed for the first time. The promise of this technique is shown by the fact that examining this morphology has given us new insight into the role of new-layer nucleation for ice-film stability [7] and allowed us to propose a mechanism for why metastable cubic ice forms at low temperatures [6]. We plan to use this capability to study the evolution of the structure of ice films as they interact with different chemical environments.

This work was supported by U. S. DOE, OBES, Division of Materials Sciences under contract DE-AC04-94AL8500.

\section{References:}

1 A. Verdaguer, G. M. Sacha, H. Bluhm, and M. Salmeron, Chem. Rev., 106, 1478 (2006).

2 V. N. Vertsner and G. S. Zhdanov, Soviet Physics - Crystallography, USA; March 1965 April 1966 10, 715 (1965).

3 I. Baker, Microscopy Research and Technique, 62, 70 (2003).

4 M. Morgenstern, J. Muller, T. Michely, and G. Comsa, Z. Phys. Chem. 198, 43 (1997).

5 A. Michaelides and K. Morgenstern, Nature Mater. 6, 597 (2007).

6 K. Thürmer and N.C. Bartelt, Phys. Rev. B 77, 195425 (2008).

7 K. Thürmer and N.C. Bartelt, Phys. Rev. Lett. 100, 186101 (2008).

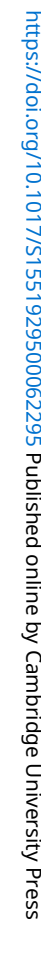




\section{See surface detail at the nanoscale like never before Magellan $^{\text {TM }}$ XHR SEM}

\section{Discover a world never seen before}

- Subnanometer resolution from $30 \mathrm{kV}$ down to $1 \mathrm{kV}$

- Surface details at low voltages you cannot see any other way

- Fast, accurate, precise analysis at higher voltages with high, stable current

- No compromises in sample size, shape, composition and preparation

- Simple and easy to use
This series of images from FEI's Magellan XHR SEM shows nanotubes imaged at $200 \mathrm{~V}$, from $10 \mathrm{k}$ to $600 \mathrm{k}$ times magnification. Note the contrast and surface detail that would not be possible on other SEMs. Images courtesy of Prof. Raynald Gauvin and Camille Probst, McGill University.

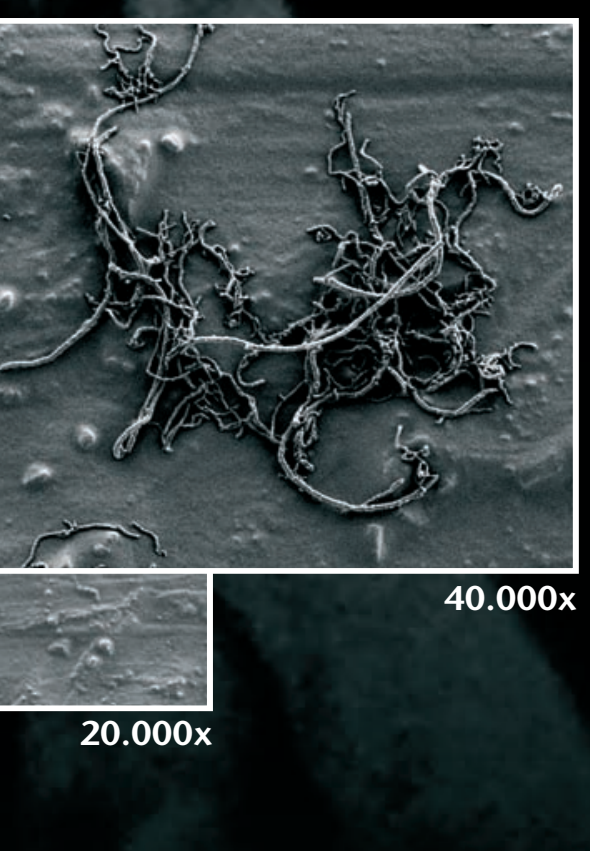

$10.000 x$

\section{Explore further at fei.com/magellan}

